\title{
Structure determination of neutral MgO clusters-hexagonal nanotubes and cages $\dagger$
}

\author{
Marko Haertelt, ${ }^{a}$ André Fielicke, ${ }^{* a}$ Gerard Meijer, ${ }^{a}$ Karolina Kwapien, ${ }^{b}$ \\ Marek Sierka $\ddagger^{* b}$ and Joachim Sauer ${ }^{b}$
}

Received 31st October 2011, Accepted 21st December 2011

DOI: $10.1039 / \mathrm{c} 2 \mathrm{cp} 23432 \mathrm{~g}$

Structural information for neutral magnesium oxide clusters has been obtained by a comparison of their experimental vibrational spectra with predictions from theory. $(\mathrm{MgO})_{n}$ clusters with $n=3-16$ have been studied in the gas phase with a tunable IR-UV two-color ionization scheme and size-selective infrared spectra have been measured. These IR spectra are compared to the calculated spectra of the global minimum structures predicted by a hybrid ab initio genetic algorithm. The comparison shows clear evidence that clusters of the composition $(\mathrm{MgO})_{3 k}(k=1-5)$ form hexagonal tubes, which confirm previous theoretical predictions. For the intermediate sizes $(n \neq 3 k)$ cage-like structures containing hexagonal $(\mathrm{MgO})_{3}$ rings are identified. Except for the cubic $(\mathrm{MgO})_{4}$ no evidence for bulk like structures is found.

\section{Introduction}

Nanotubes, rods, or cages are fascinating structural motifs and for instance by changing their aspect ratio, ${ }^{1}$ adsorption of other molecules ${ }^{2,3}$ or filling, ${ }^{4}$ their electronic, optical or chemical properties may be tuned and adapted to possible applications, e.g., in nanodevices. ${ }^{5-7}$ Elongated structures like nanotubes or nanorods can be formed, apart from carbon, from many different other substances like elemental compounds (including metals), oxides, nitrides, and carbides ${ }^{8}$ and also magnesium oxide. Bundles of $\mathrm{MgO}$ nanotubes with length up to several tens of $\mu \mathrm{m}$ can be made by simple thermal evaporation methods. ${ }^{9-11}$ Though the macroscopic shape of these tubes may not easily fit into the picture of a cubic crystal, on an atomic scale the cubic motif of the bulk $\mathrm{MgO}$ in its rocksalt structure is still retained. For nanoscaled clusters, however, we observe a different behavior.

Already the first experiments on $\mathrm{MgO}$ clusters by multiple photon laser ionization mass spectrometry ${ }^{12}$ revealed that in addition to the cubic motif tubular structures formed by stacking hexagonal $(\mathrm{MgO})_{3}$ units - hexagonal nanotubes - may be present. A number of theoretical studies have followed these

${ }^{a}$ Fritz-Haber-Institut der Max-Planck-Gesellschaft, Faradayweg 4-6, D-14195 Berlin, Germany.E-mail: fielicke@fhi-berlin.mpg.de

${ }^{b}$ Institut für Chemie, Humboldt-Universität zu Berlin,

Unter den Linden 6, D-10099 Berlin, Germany.

E-mail: marek.sierka@chemie.hu-berlin.de

$\dagger$ Electronic supplementary information (ESI) available: Calculated vertical ionization energies, cubic sub-structures for $(\mathrm{MgO})_{15}$ and coordinates for all structures. See DOI: 10.1039/c2cp23432g

$\ddagger$ Present address: Computational Material Science, Institut für Materialwissenschaft und Werkstofftechnologie, Friedrich-Schiller-Universität Jena Löbdergraben 32, D-07743 Jena, Germany. experimental investigations, most applying simple ion (pair) potentials ${ }^{13-17}$ sometimes in combination with global structure optimization schemes. ${ }^{18-21}$ Such simulations applying parameterized potential functions predict two different structural types depending on the ionic charges: a cubic motif for full formal charges and hexagonal tubes and cages ${ }^{18}$ for smaller charges. Hexagonal tubes have been identified by a genetic algorithm $^{20}$ as the putative global minima for clusters with $(\mathrm{MgO})_{3 k}, k=1-5$. For other cluster compositions often more cube-like structures are predicted, but there is no common agreement on their structures in the literature yet. Quantum chemical methods ${ }^{22-27}$ in combination with global optimization approaches have to our knowledge not yet been systematically applied to neutral $\mathrm{MgO}$ clusters of different sizes. Only for the alkaline halide clusters $(\mathrm{LiF})_{n}$, which are assumed to behave very similar to $\mathrm{MgO}$ clusters, ${ }^{15}$ simulated annealing at an $a b$ initio level has been performed for $n=1-8 .{ }^{28}$ Experimental data to benchmark the theoretical predictions are highly desirable especially for larger systems, but spectroscopic information is up to date rather limited.

For determining the structure of strongly bound gas-phase clusters the combination of IR excitation with mass spectrometric detection has been proven to be very successful. ${ }^{29}$ Obtaining IR spectra of neutral metal oxide clusters, however, is challenging. Matrix isolation spectroscopy has been used to study very small $\mathrm{MgO}$ species, in particular $\mathrm{OMgO}$ and $(\mathrm{MgO})_{2} \cdot{ }^{30}$ For larger clusters in the gas phase, starting with $(\mathrm{MgO})_{15}$ up to $(\mathrm{MgO})_{168}$ IR resonance enhanced multiple photon ionization (IR-REMPI) spectra ${ }^{31}$ have been reported and very similar IR spectra for all sizes have been found. Because of the vicinity of the observed bands to the surface phonon modes the cubic motif was suggested as being already 
present at smaller sizes. However, quantum chemical calculations were missing at that time, making a definite structural assignment impossible. In such gas-phase experiments the neutral clusters can be investigated without any perturbation from the environment. However, the absorption of several hundred IR photons needed to induce IR-REMPI requires a high IR fluence and the high internal energies necessary for the thermionic electron emission usually lead to broadening and shifting of spectral lines, making the spectra less informative.

For this reason more evolved techniques have been developed, which have so far mainly been used to study ionic metal oxide clusters. IR multiple photon dissociation of the cluster itself or the dissociation of more weakly bound messenger complexes has been used to measure vibrational spectra of metal oxide cluster ions. ${ }^{32-37}$ Messenger tagging has recently also been used by us to obtain IR spectra of small cationic $(\mathrm{MgO})_{2-7}^{+}$clusters. ${ }^{38}$ For these, surprising structures have been identified, which are different from the theoretically predicted ones of the neutral $\mathrm{MgO}$ clusters.

Compared to the experiments on ionic clusters, the mass spectrometric investigation of neutral species requires an additional ionization step. For this, usually UV single photon ionization is used to avoid excessive fragmentation of the clusters. However, direct investigation of neutral metal oxides with such a technique is hampered by the large ionization energies (IE $>8 \mathrm{eV}$ ) typical for oxygen rich metal oxide clusters, as no 'simple' high fluence laser sources are available with a photon energy above $8 \mathrm{eV}$.

The technique used in this paper makes direct use of the rather high IEs of the $\mathrm{MgO}$ clusters and does not rely on any messenger which might perturb the cluster's structure. A tunable IR-UV two-color ionization scheme ${ }^{39}$ recently used to obtain far-IR spectra of neutral silicon clusters, ${ }^{40}$ for instance, was applied to obtain vibrational spectra of $(\mathrm{MgO})_{n}$ clusters containing between 3 and $16 \mathrm{MgO}$ units. Thereby, this study closes the gap between the matrix isolation studies for very small $\mathrm{MgO}$ clusters and the IR-REMPI investigations on large $\mathrm{MgO}$ clusters. The structures of $\mathrm{MgO}$ clusters are assigned by comparison with calculated IR spectra for low energy isomers located using an ab initio global optimization employing genetic algorithm. Our approach delivers detailed insights into the structural evolution of small neutral $\mathrm{MgO}$ clusters and reveals dramatic structural differences between $\mathrm{MgO}$ in the bulk phase and at the nanoscale.

\section{Methods}

\subsection{Experimental method}

The experimental setup has been extensively described before, ${ }^{33,41}$ therefore the focus of this section will be primarily on the ionization scheme used to record the IR spectra of the neutral metal oxide clusters. $\mathrm{MgO}$ clusters are made by laser ablation from a target of isotopically enriched ${ }^{26} \mathrm{Mg}(99.4 \%$, Oak Ridge National Laboratory) in a gas pulse of $1 \%$ oxygen in helium. The clusters are cooled to $100 \mathrm{~K}$ in a thermalization channel attached to the source. Expansion into vacuum takes place and a combination of a skimmer and a flat aperture with an opening of $1 \mathrm{~mm}$ is used to shape the beam. A voltage applied

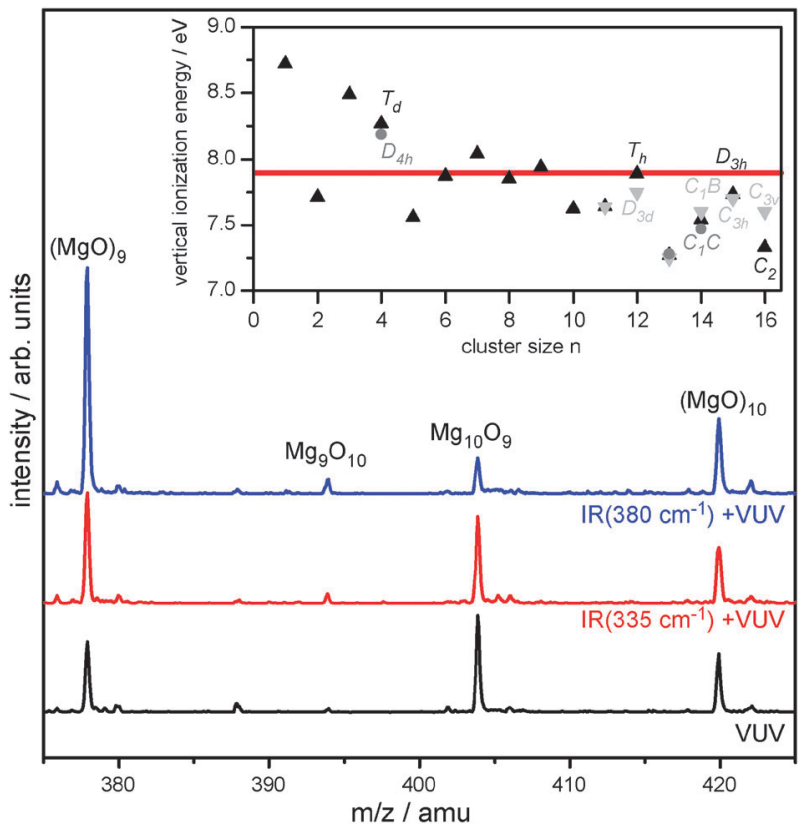

Fig. 1 Time-of-flight mass spectra obtained under different ionization conditions. Upon irradiation with just $7.87 \mathrm{eV}$ photons, only weak signals are observed, which significantly increase for stronger bound systems when IR radiation, resonant with a vibrational transition, is added. The inset shows the calculated vertical IEs of the low energy structures obtained from the global optimization (see also ESI $\dagger$ ). The red line indicates the photon energy of the $\mathrm{F}_{2}$ excimer laser $(7.87 \mathrm{eV})$.

to the aperture removes all ions from the beam. The remaining neutral cluster distribution is analyzed with a reflectron timeof-flight mass spectrometer by using an unfocussed $\mathrm{F}_{2}$ excimer laser (7.87 eV photon energy) for ionization. Small peaks are observed at the positions for the one-to-one stoichiometric magnesium oxide clusters, e.g. $\operatorname{Mg}_{n} \mathrm{O}_{m}$ with $n=m$, as well as for systems deviating slightly from this stoichiometry, e.g. $n=m+2, m \pm 1$, see also Fig. 1. Experimental values of the ionization energies (IEs) for such systems are not available. We therefore refer here to the calculated IEs for the low lying isomers discussed later. For systems with $n=m$ they are around $8 \mathrm{eV}$, see the inset in Fig. 1. From this, one might already expect that these clusters are not very efficiently ionized at a photon energy of $7.87 \mathrm{eV}$ of the $\mathrm{F}_{2}$ laser. Indeed, the weak mass spectrometric signals observed confirm the calculations of the IEs qualitatively. The residual signals can be assigned to the hot fraction of the thermal cluster distribution, where the internal energy in combination with the UV photon energy is sufficient for ionization. The internal energy distribution for $(\mathrm{MgO})_{9}$, for example, is shown in Fig. 2, and was derived with the help of the Beyer-Swinehart algorithm ${ }^{42}$ by assuming a Boltzmann distribution close to the source temperature of $100 \mathrm{~K}$. The majority $(\sim 40 \%)$ of the clusters are in the vibrational ground state, and only a tiny fraction of $\sim 1 \%$ have an internal energy greater than $0.1 \mathrm{eV}$, and $10^{-4 \%}$ an energy of more than $0.25 \mathrm{eV}$.

The ionization efficiency usually follows a $S$-curve behavior as a function of excitation energy, with a slope depending on the Franck-Condon overlap between neutral and cationic structure. A rise in the internal energy of the clusters could 


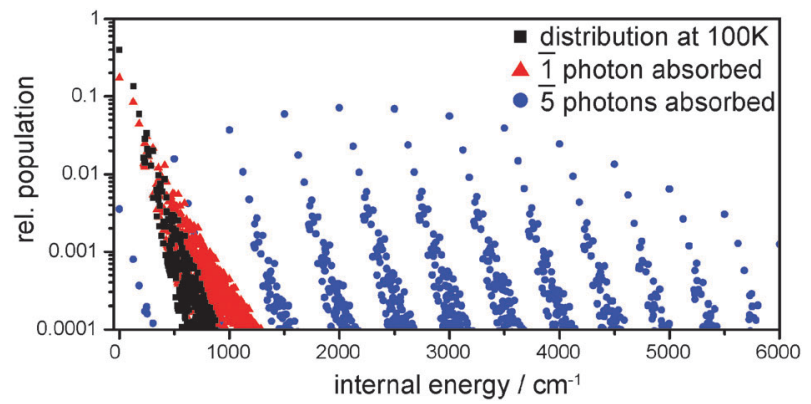

Fig. 2 Distribution of the internal energy for $(\mathrm{MgO})_{9}$ at $100 \mathrm{~K}$ (black squares) and after an average absorption of 1 and 5 photons at $500 \mathrm{~cm}^{-1}$ shown as red arrows and blue dots, respectively.

therefore increase the amount of ionizable clusters. ${ }^{43}$ This can be used to obtain cluster size specific IR spectra by resonant excitation with IR light. For this purpose the cluster beam is overlapped with the focussed IR radiation from the Free Electron Laser for Infrared eXperiments (FELIX). ${ }^{44}$ The molecular and the IR beam are counter-propagating and the clusters get irradiated before they pass through a $1 \mathrm{~mm}$ aperture in front of the extraction region of the mass spectrometer. The IR beam is weakly focussed through the aperture, such that all clusters passing through it are exposed to the $\sim 7 \mu$ s long macropulse of IR radiation. Similar to IR-REMPI or multiple photon dissociation, during this macropulse, the clusters can undergo several absorption and internal vibrational redistribution cycles, which increase the internal energy. As this resonant excitation takes place a few $\mathrm{cm}$ before the extraction region, and thereby approximately $30 \mu$ s before they get ionized with the additional UV photon, the process is completely sequential, and direct IR-UV two photon processes do not play a role.

The increase of the relative amount of 'hot' clusters can be modeled by a simple Monte-Carlo simulation with a fixed absorption probability. For $\mathrm{C}_{60}$ it has been found before that such an approach can give quantitatively similar results to a numerical modeling of the vibrational ladder climbing considering explicitly also the effects of (cross-) anharmonicities. ${ }^{45}$ The internal energy distribution for the average absorption of a single photon and five photons is shown in Fig. 2 assuming an absorption probability of $p=0.01$ and a photon energy of $500 \mathrm{~cm}^{-1}$ for $(\mathrm{MgO})_{9}$. The amount of clusters with an internal energy above $0.25 \mathrm{eV}$ is rising dramatically to $2 \%$ for a $\overline{1}$ photon absorption and to $67 \%$ for $\overline{5}$ photon absorption.

The cluster-size specific IR spectra are obtained by recording on alternating shots mass spectra with (intensity $I$ ) and without FELIX irradiation $\left(I_{0}\right)$ and by scanning the IR wavelength. A resonant absorption typically expresses itself as significant increase in the ion intensity in the time-of-flight spectrum. An example is shown in Fig. 1. Upon irradiation at $380 \mathrm{~cm}^{-1}$ the observed rise in intensity for $(\mathrm{MgO})_{9}$ amounts to a factor of three. Fig. 1 shows also the competing process to ionization; more loosely bound species tend to fragment by the IR radiation and cannot be detected with the mass spectrometer. The IR absorption spectrum of such species can be recorded by monitoring the depletion of the mass spectrometric signal. Almost all species with $\mathrm{Mg}_{n} \mathrm{O}_{m}$ with $n>m$ show this behavior, while systems with $n=m$ and $m>n$ can be studied with the ionization technique. A macropulse energy of $\sim 16 \mathrm{~mJ}$ was used in the whole range, with an approximate beam diameter of $1 \mathrm{~mm}$ at the aperture. Relative absorption spectra are derived from the relative enhancements of the ion signal by $\left(I-I_{0}\right) / I_{0}$ normalized by the photon flux.

\subsection{Theoretical method}

DFT calculations are performed using the TURBOMOLE program package. ${ }^{46}$ For the global structure optimizations the hybrid $a b$ initio genetic algorithm ${ }^{47}$ is applied along with the B3-LYP exchange-correlation functional ${ }^{48,49}$ and triple-zeta valence plus polarization basis set ${ }^{50-52}$ for all atoms. To speed up the DFT calculations we use the multipole accelerated resolution of an identity (MARI-J) method $^{53}$ along with the corresponding TZVP auxiliary basis sets. All minima were verified by vibrational analysis. Frequency calculations are performed within the harmonic approximation using analytical second derivatives. IR spectra are simulated using unscaled vibrational frequencies and intensities calculated in the harmonic approximation from analytical derivatives of the dipole moment. The overall agreement between simulated and observed IR spectra is very good in the present case. For this reason we did not attempt to scale the calculated IR frequencies. The spectra are convoluted using a Gaussian line shape function with a half-width of $10 \mathrm{~cm}^{-1}$. The effect of zero-point vibrational energies (ZPVE) on energetic ordering of different isomers was found to be negligible. Therefore, the here reported relative energies do not include ZPVE contributions.

\section{Results and discussion}

Vibrational spectra of $(\mathrm{MgO})_{n}$ clusters have been measured in the $200-1700 \mathrm{~cm}^{-1}$ region. This covers the range where the characteristic vibrations of metal oxide clusters are expected, and also makes it possible to identify molecular dioxygen species and how they are bound to the cluster. The different possible dioxygen ligands can be unambiguously identified by their IR spectra. The physisorbed oxygen molecule absorbs at around $1550 \mathrm{~cm}^{-1}$, while the superoxo species has a characteristic vibration at around $1100 \mathrm{~cm}^{-1}$. The peroxo species is more difficult to assign as its band is around $700-800 \mathrm{~cm}^{-1}$, where also the stretches of metal-oxygen units are found. For none of the species considered in this article modes above $1000 \mathrm{~cm}^{-1}$ are seen, which makes the presence of physisorbed dioxygen as well as the superoxo species unlikely. This is quite different from the cationic $\mathrm{MgO}$ clusters investigated recently. ${ }^{38}$ There, some of the measured bands have been assigned to isomers containing dioxygen species. As no additional structural information is contained in the range above $1000 \mathrm{~cm}^{-1}$, the experimental spectra are here only reported for the 200-1000 $\mathrm{cm}^{-1}$ range. The experimental spectra for the $(\mathrm{MgO})_{n}$ clusters with $n=3-16$ together with a comparison to the results of the calculations are shown in Fig. 3-5. An overview of all structures can be found in Fig. 7. For most sizes very distinct IR spectra have been recorded. In several cases a comparably low number of bands is found, which can be an indication for rather symmetric structures. For all sizes above $n>5$ a very intense band is observed at around $700 \mathrm{~cm}^{-1}$, which seems to be saturated and power broadened. For lower 
frequencies saturation effects do not seem to play a major role for the quality of the spectra. The observed bands can be as narrow as $<10 \mathrm{~cm}^{-1}$ full width at half maximum (FWHM).

\subsection{The hexagonal tubes- $(\mathrm{MgO})_{3 k}$ clusters}

The vibrational spectra for clusters with $(\mathrm{MgO})_{3 k}$ composition $(k=1-5)$ are shown in Fig. 3. Already at the first glance large similarities between the spectra of different sizes become apparent. This may indicate a similar building principle for the clusters based on the $(\mathrm{MgO})_{3}$ unit. Indeed, previous theoretical studies, initiated by the observation of magic peaks in the mass spectra, ${ }^{12}$ predicted for these sizes with $n=3 \times k$ the presence of hexagonal tube structures formed by stacking $k(\mathrm{MgO})_{3}$ hexagons. Also our global optimization scheme identifies the hexagonal tubes as the global minima for these sizes. Only for $(\mathrm{MgO})_{3 \times 4}$ a cage-like structure is found $1.8 \mathrm{~kJ} \mathrm{~mol}^{-1}$ lower in energy. The calculated linear absorption spectra for these low energy structures and the experimental spectra are compared in Fig. 3.

For all sizes the spectrum of the hexagonal ring or tube structure shows a very good match to the experimental spectrum.

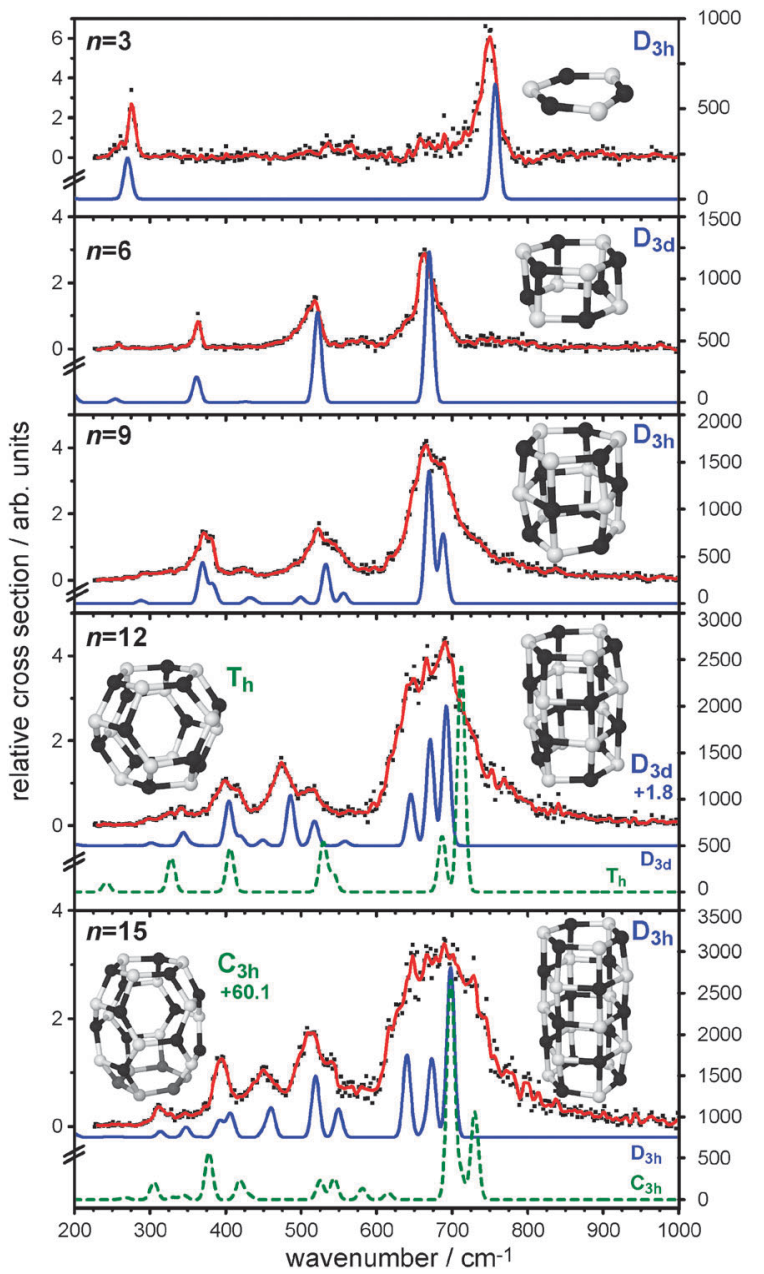

Fig. 3 Vibrational spectra of $(\mathrm{MgO})_{3 k}$ clusters with $k=1-5(3 k=n)$ and comparison to calculated linear absorption spectra. The measured data points are shown as dots and a five-point-running average (solid red line) is added to guide the eye. Some of the theoretical spectra are vertically shifted for clarity. Relative energies of different isomers are given in $\mathrm{kJ} \mathrm{mol}^{-1}$ and do not include zero point vibrational energy.
For the hexagonal ring $(\mathrm{MgO})_{3 \times 1}$ and the small tubes with $(\mathrm{MgO})_{3 \times 2}$ and $(\mathrm{MgO})_{3 \times 3}$ there is little doubt about the assignment, as the putative calculated minima also explain finer details of the measurements, e.g., the weak band for $(\mathrm{MgO})_{3 \times 2}$ at $240 \mathrm{~cm}^{-1}$. The spectrum allows a clear assignment to a single isomer, whereas for its cationic counterpart, $(\mathrm{MgO})_{3 \times 2}{ }^{+}$, theory predicts three low lying isomers with their energetic ordering strongly depending on the theoretical methods used. None of them, nor a simple combination, completely explains the very complex experimental spectrum. ${ }^{38}$ The structure now assigned for the neutral cluster was also one of these three low energy isomers found for the cation. For the next larger size, $(\mathrm{MgO})_{3 \times 4}$, a cage with $T_{\mathrm{h}}$ symmetry is predicted lower in energy than the tube, but its spectrum does not fit as well with the experimental spectrum, which can be fully explained by the tube structure. Also inclusion of zero point vibrational energy differences does not alter the order of the two isomers. The energy difference even slightly increases from 1.8 to $3.2 \mathrm{~kJ} \mathrm{~mol}^{-1}$. The predicted IEs for the two isomers are $7.89 \mathrm{eV}$ for the cage and $7.75 \mathrm{eV}$ for the tube structure. As the IR spectroscopic technique used here exploits relative enhancements in the ionization efficiency of the systems, it is more sensitive to the isomers with the lower IE. Therefore, the additional presence of the cage isomer cannot be completely ruled out.

For the $(\mathrm{MgO})_{3 \times 5}$ cluster the hexagonal tube is again the lowest energy structure and its spectrum nicely fits the experimental data. The next higher isomer, energetically separated by $60.1 \mathrm{~kJ} \mathrm{~mol}^{-1}$, is a cage of $C_{3 \mathrm{~h}}$ symmetry. It shows large similarities with the cage structure calculated as the global minimum of $(\mathrm{MgO})_{3 \times 4}$ and can be formed by adding an additional hexagonal ring to the $(\mathrm{MgO})_{3 \times 4}$ cage.

Although the IR spectra for all tubes appear to have many common features, the characteristic vibrations of the tubular structure like concerted movements of the layers, breathing modes or even kinks of the whole tube are either not IR active or show only very low IR intensities. The modes of high IR intensities observed in the experiment are strongly delocalized and involve the movements of almost all atoms.

\subsection{Small non-tubular MgO clusters}

Besides the $(\mathrm{MgO})_{3 k}$ clusters adopting the perfect hexagonal tube structures also the intermediate sizes $(n \neq 3 k)$ turn out to have interesting structures, most of them strongly deviating from the bulk. The experimental spectra and the comparison to the theoretical predictions for these non-tubular clusters containing up to ten $\mathrm{MgO}$ units are shown in Fig. 4. Except for the smallest size $(n=4)$, the experimental findings can be explained by the calculated IR spectrum of a single isomer, the predicted global minimum.

For $(\mathrm{MgO})_{4}$ theory identifies a cube with $T_{\mathrm{d}}$ symmetry as the global minimum. From all sizes studied, it is the only one, which has a bulk-like structure. The cube has four IR active modes at $328,532,566$, and $570 \mathrm{~cm}^{-1}$, which are up to triply degenerate. The two most intense experimental features are reproduced by this structure, but the bands experimentally observed at $\sim 820 \mathrm{~cm}^{-1}$ and $280 \mathrm{~cm}^{-1}$ cannot be explained by this isomer. This $280 \mathrm{~cm}^{-1}$ band and also the highest frequency one might be explained by the 8-membered ring isomer of $D_{4 \mathrm{~h}}$ symmetry, $64.2 \mathrm{~kJ} \mathrm{~mol}^{-1}$ above the global minimum. 


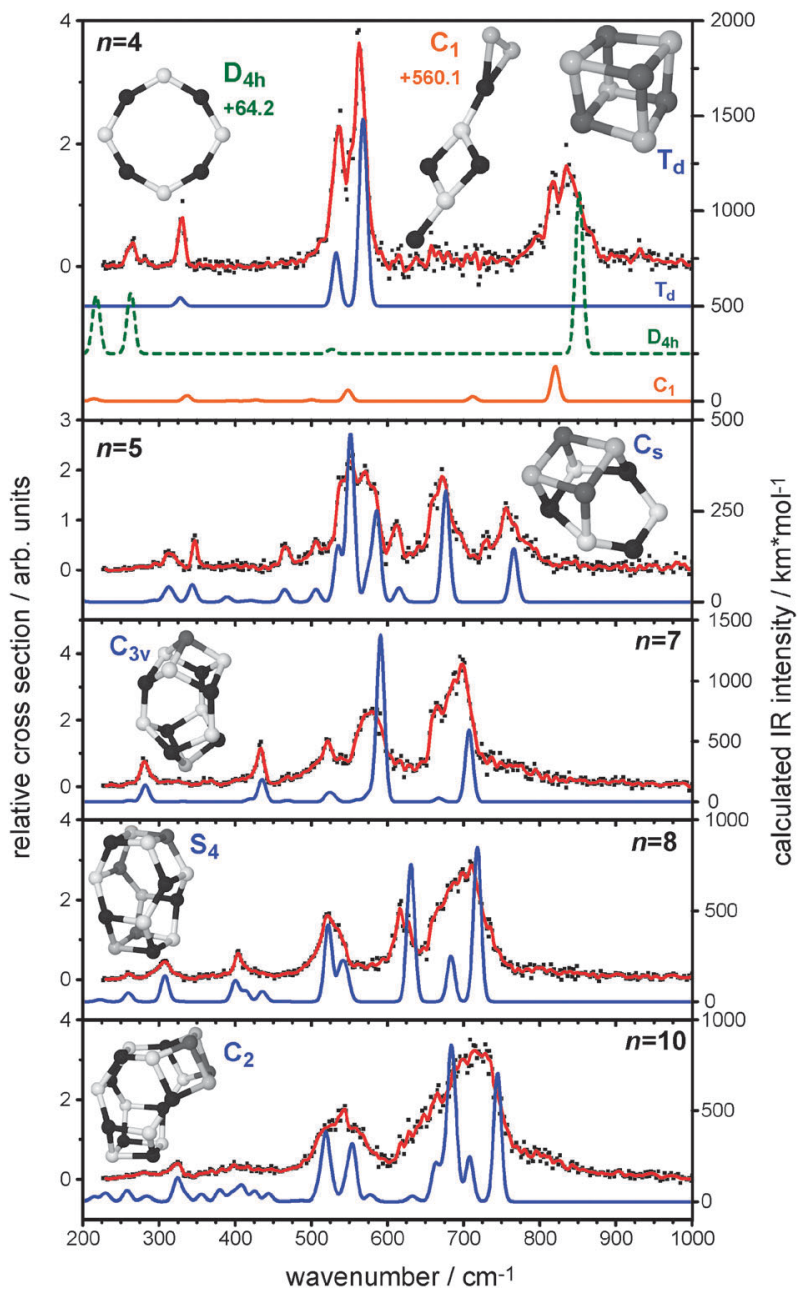

Fig. 4 Measured and calculated IR spectra of smaller $(\mathrm{MgO})_{n}$ clusters with $n=4,5,7,8,10$. Comparison with theory shows that more cage-like structures are observed, and that the hexagonal ring is the main building block in the observed structures.

The calculation predicts a vibration at $852 \mathrm{~cm}^{-1}$, which would partially explain the broad mode at around $820 \mathrm{~cm}^{-1}$. But it seems to have a finer structure, with sharper features at 817 and $834 \mathrm{~cm}^{-1}$, which might indicate the presence of even more isomers.

At such frequency also peroxo modes are found and also for the cations such species were suspected as being additionally present in the molecular beam. For instance for the $(\mathrm{MgO})_{4}^{+}$ and $(\mathrm{MgO})_{7}^{+}$clusters features at such frequencies were observed, which could not be assigned to belong to a specific isomer. ${ }^{38}$ To understand the dioxygen binding to neutral, but also cationic clusters, it has been investigated how molecular oxygen binds to $\mathrm{Mg}_{4} \mathrm{O}_{2}$. Surprisingly, superoxo complexes form instead of peroxo complexes. These superoxo complexes are high in energy, but may not easily relax to the global minimum. The spectrum of such a structure is also shown in Fig. 4. The $\mathrm{O}-\mathrm{O}$ bond distance is $1.36 \AA$, quite typical for such a species. The superoxo mode is found at $1149 \mathrm{~cm}^{-1}$ with a calculated IR cross section of only $0.19 \mathrm{~km} \mathrm{~mol}^{-1}$. This is much weaker than for any other feature observed, which would explain its absence in the experiment. Besides the superoxo mode also all other features are comparably weak.
The most intense feature is predicted at $820 \mathrm{~cm}^{-1}$, the position where the peroxo mode was expected. This position agrees very well with the first sharper peak in the $820 \mathrm{~cm}^{-1}$ feature. Also the weaker mode at $336 \mathrm{~cm}^{-1}$ is only $5 \mathrm{~cm}^{-1}$ higher than in the experiment. A similar complex would also explain the experimental IR spectrum of the $(\mathrm{MgO})_{7}^{+}$cluster, as this showed absorptions at 780 and $1095 \mathrm{~cm}^{-1}$.

The global minimum structure of $(\mathrm{MgO})_{5}$ is completely different from the one of the cationic cluster. There, an open book-like structure was observed. For the neutral a more compact structure is found that carries both the cubic as well as the hexagonal ring motifs. It can either be seen as being formed by adding a $(\mathrm{MgO})_{2}$ unit to the $(\mathrm{MgO})_{3}$ hexagonal ring, or by adding a $\mathrm{MgO}$ bridge to the $(\mathrm{MgO})_{4}$ cluster.

For the larger sizes the main structural motif is based on the $(\mathrm{MgO})_{6}$ cluster. The structure of $(\mathrm{MgO})_{7}$ is cage-like with $C_{3 \mathrm{v}}$ symmetry and seems first to have nothing in common with most of other structures. However, it can be seen as a $(\mathrm{MgO})_{6}$ unit, where the hexagons became buckled after adding $\mathrm{O}$ and $\mathrm{Mg}$ atoms to the two open ends of the tube. For the larger sizes, the $(\mathrm{MgO})_{6}$ cluster motif is more evident. The $(\mathrm{MgO})_{8}$ cluster, for example, contains two of these units, fused at $90^{\circ}$. Another way to describe the structure is by adding a $(\mathrm{MgO})_{2}$ unit to the side of the $(\mathrm{MgO})_{6}$ cluster. The structure of $(\mathrm{MgO})_{10}$ is also built up by this principle. Its core is formed by a $(\mathrm{MgO})_{8}$ cluster that is capped with a $(\mathrm{MgO})_{2}$ unit. The symmetry changes from $S_{4}$ for $(\mathrm{MgO})_{8}$ to $C_{2}$ for $(\mathrm{MgO})_{10}$.

The identified global minima in the range up to $(\mathrm{MgO})_{8}$ and for the tubes up to $k=5$ are identical to the predictions for the alkaline halide system $\mathrm{LiF}^{28,54}$ For $(\mathrm{LiF})_{4}$ the barrier landscape was also calculated and it was predicted that the cube and the octahedron ring could both be observed in the experiment. ${ }^{28}$ It appears that these two isomers are present also in the case of $(\mathrm{MgO})_{4}$, but here even a third isomer needs to be considered.

Also the most extensive calculation on $\mathrm{MgO}$ using a genetic algorithm with a Born-Mayer potential and post-optimization with DFT $^{20}$ predicted structures consistent with our findings for all tubular structures $(\mathrm{MgO})_{3 k}$ as well for the other sizes up to $(\mathrm{MgO})_{7}$. For $(\mathrm{MgO})_{8}$ and $(\mathrm{MgO})_{10}$, however, we identify quite different structures.

\subsection{Large $(\mathrm{MgO})_{n}$ clusters $(n>10)$-between imperfect tubes and cages}

Whereas for the perfect hexagonal tubes up to rather large sizes $(n=15)$ the quality of the experimental spectra is sufficient to confirm the presence of the predicted putative global minima found by the genetic algorithm, this becomes more difficult for the large non-tubular clusters. The number of bands in the vibrational spectra seems to increase and leads to the observation of rather broad absorptions ( $c f$. Fig. 5). At the same time the potential energy surface for such large species usually becomes more complicated and many isomers can lie close in energy. Therefore, also in the experiment multiple isomers might be observed. In addition, the ionization energy drops for larger sizes, yielding a significant ionization probability already for colder clusters. This reduces the relative amount of the IR induced enhancement of the ionization efficiency and makes, eventually, the application of our 


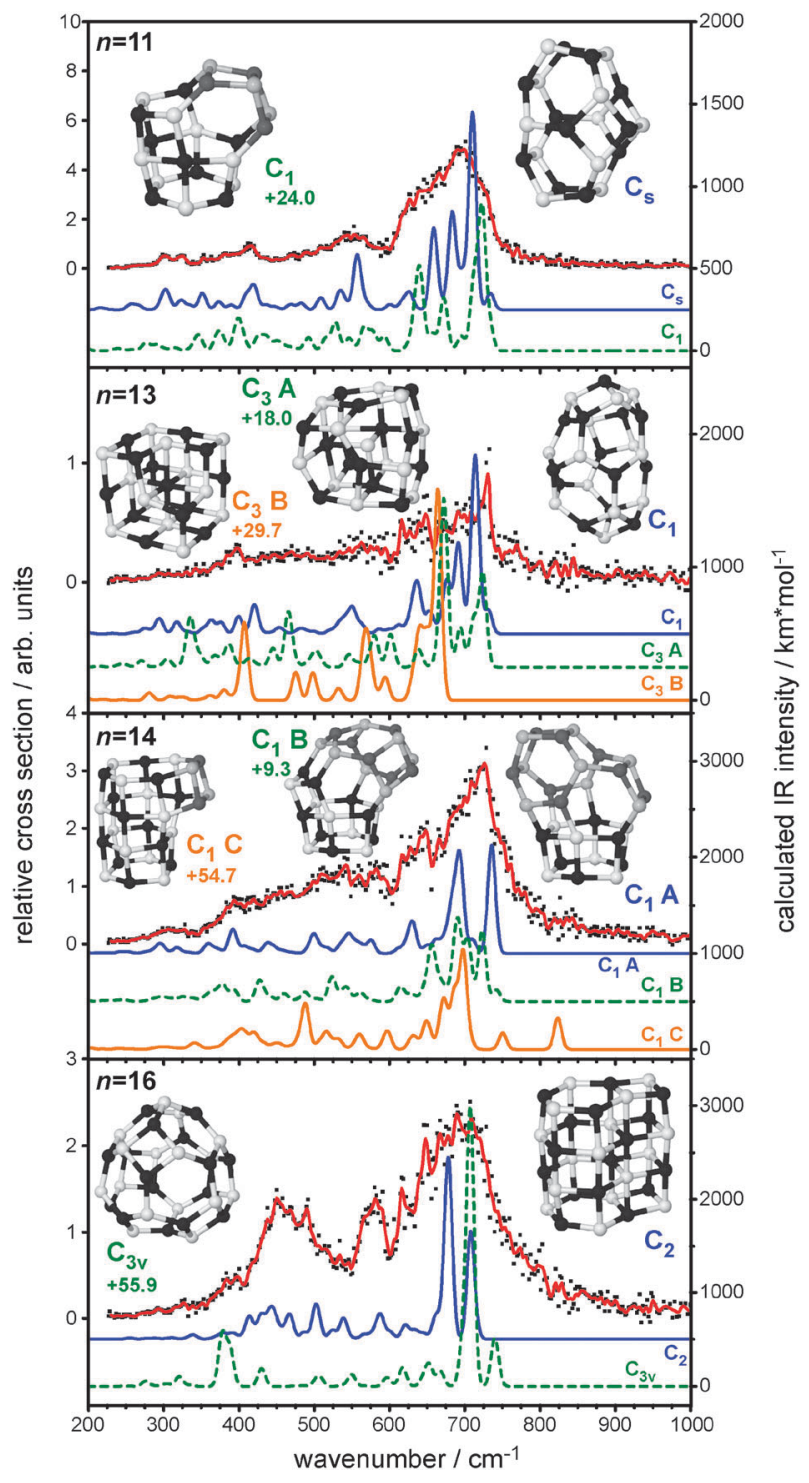

Fig. 5 Measured and calculated IR spectra for several isomers of $(\mathrm{MgO})_{11},(\mathrm{MgO})_{13},(\mathrm{MgO})_{14}$, and $(\mathrm{MgO})_{16}$.

spectroscopic technique unfeasible. In particular the $(\mathrm{MgO})_{13}$ cluster seems to be affected by this problem. The calculated IE for the identified global minimum is only $7.27 \mathrm{eV}$, which is $0.6 \mathrm{eV}$ below the photon energy of the ionization laser. This calculation is confirmed by the high intensity observed in the time-of-flight mass spectrum, which is almost a factor of two larger than the neighboring $(\mathrm{MgO})_{12}$ signal and a factor of three larger than that of $(\mathrm{MgO})_{14}$ and, indeed, the calculated IEs of $(\mathrm{MgO})_{12}$ and $(\mathrm{MgO})_{14}$ are clearly higher. Nevertheless, some small IR induced increase in the ion intensity is observed, leading to an IR spectrum of relatively low signal-to-noise level.

The genetic algorithm identifies for $(\mathrm{MgO})_{11}$ a cage-like structure of $C_{\mathrm{s}}$ symmetry that shows some similarity with the $(\mathrm{MgO})_{8}$ structure. All main features in the experimental spectrum can be explained by this structure, in particular the features at about 300,410 , and $540 \mathrm{~cm}^{-1}$. Also the broad band at $700 \mathrm{~cm}^{-1}$ is reproduced by a multiplet of lines. Another isomer, which is closer to a tubular structure, is only $24 \mathrm{~kJ} \mathrm{~mol}^{-1}$ higher in energy. Although the agreement of its spectrum with the experiment is not bad, the band positions do not fit as well as for the global minimum. This becomes apparent especially in the lower frequency part of the spectrum. Also for $(\mathrm{MgO})_{13}$ a cage structure is identified as the putative global minimum. Some of the calculated modes are reproduced in the experimental spectrum, but the lower signal-to-noise level precludes a definite structural assignment. The next higher energy structures can be seen as somewhat distorted fragments of the $\mathrm{MgO}$ bulk structure. Both are close in energy with 18 and $29 \mathrm{~kJ} \mathrm{~mol}^{-1}$ above the global minimum. Their spectral features could explain parts of the measured spectrum. Therefore, the presence of these additional isomers cannot be completely ruled out.

The experimental spectrum of $(\mathrm{MgO})_{14}$ could be explained by the presence of either of the two lowest energy isomers (A and B) or a mixture of them, as shown in Fig. 5. Already the supposed minimum structure $\mathbf{A}$ explains most of the experimental features, in particular in the $250-600 \mathrm{~cm}^{-1}$ range. All bands have comparable IR intensities and overlap, thereby forming a very broad absorption band with little structure. Only in the $600-750 \mathrm{~cm}^{-1}$ range sharper features are seen. The one at $640 \mathrm{~cm}^{-1}$ could either be explained by the putative global minimum $\mathbf{A}$, which would also account for a small drop in the experimental intensity at $600 \mathrm{~cm}^{-1}$, or by the second lowest structure $\mathbf{B}$, which is $9.3 \mathrm{~kJ} \mathrm{~mol}^{-1}$ higher in energy. This isomer might also explain the most intense feature at $700 \mathrm{~cm}^{-1}$, although the calculated IR intensity does not completely fit. Both isomers show large similarities with the $C_{1}$ structure of the $(\mathrm{MgO})_{11}$ cluster. The third lowest structure, isomer $\mathbf{C}$, which is $54.7 \mathrm{~kJ} \mathrm{~mol}^{-1}$ above the ground-state, does not seem to be observed in the experiment, as the band at $820 \mathrm{~cm}^{-1}$ is missing.

The structure of the global minimum for $(\mathrm{MgO})_{16}$ assigned by the genetic algorithm contains the hexagonal tube motif of $(\mathrm{MgO})_{12}$ as well as a cubic stack. Even for such a large size there is still decent agreement found between the calculated linear absorption spectrum and the experimental spectrum, although the relative band intensities fit less well. In particular the features at 490 and $590 \mathrm{~cm}^{-1}$ can be explained by this structure. The additional presence of the second lowest isomer, a cage-structure, $55.9 \mathrm{~kJ} \mathrm{~mol}^{-1}$ above the minimum, cannot be completely ruled out. The feature at $390 \mathrm{~cm}^{-1}$ and the high-energy shoulder of the $700 \mathrm{~cm}^{-1}$ band might originate from this isomer.

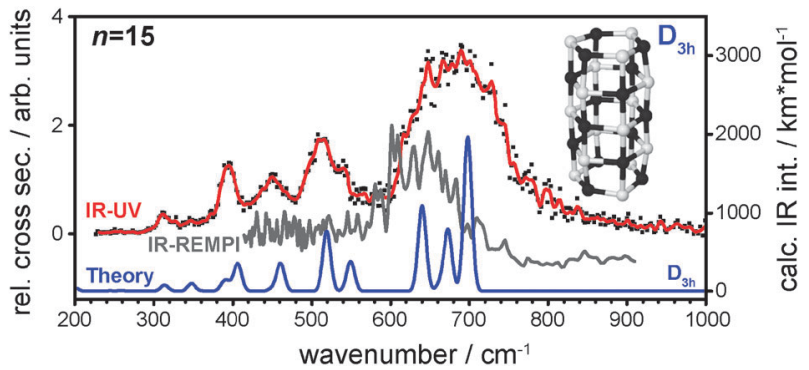

Fig. 6 Comparison of the IR-REMPI spectrum (grey line) of $(\mathrm{MgO})_{15}$ of natural isotopic distribution taken from ref. 31, with the IR-UV two-color ionization spectrum of $\left({ }^{26} \mathrm{MgO}\right)_{15}$. 

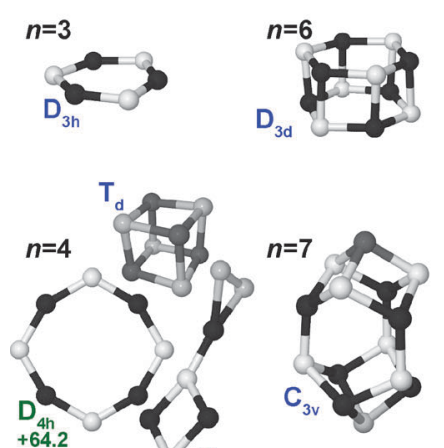
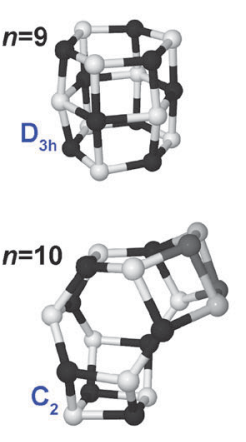

$n=12$
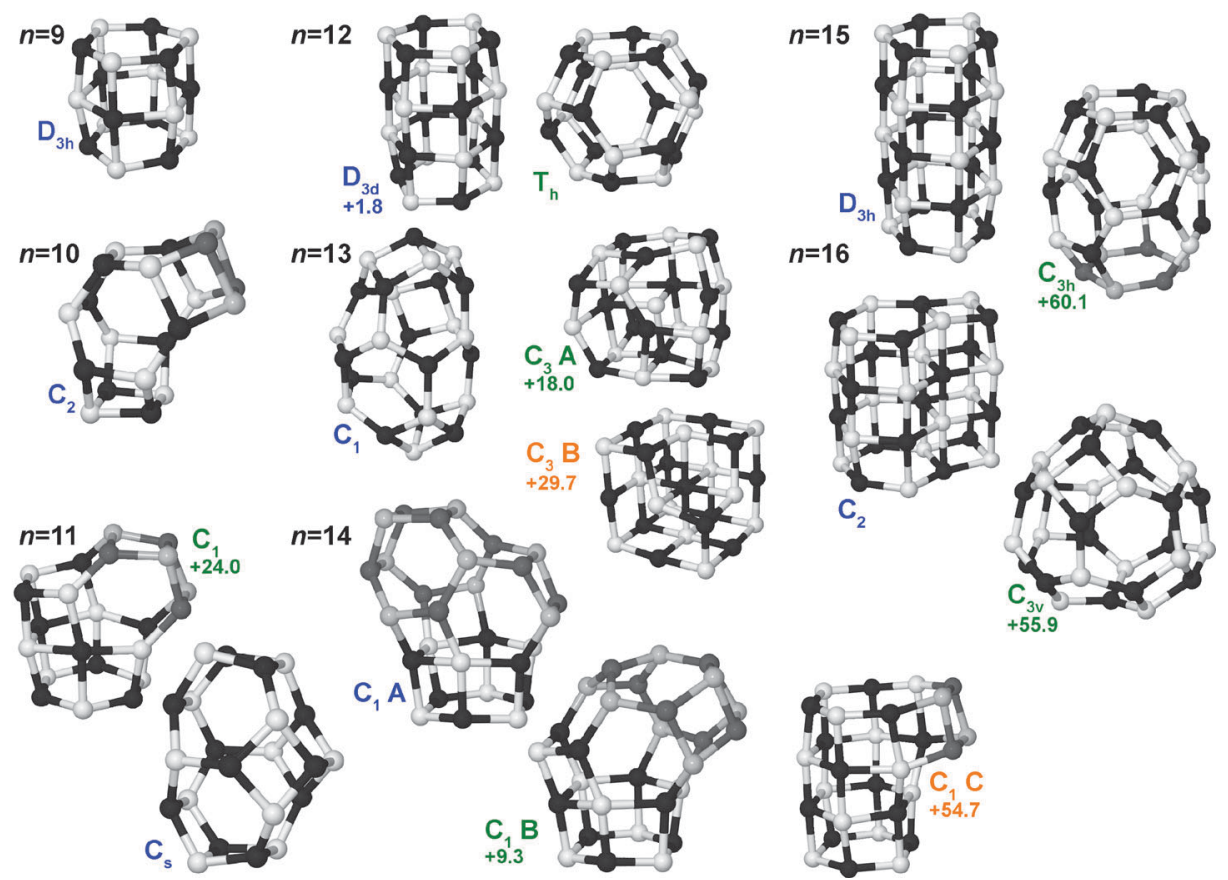

Fig. 7 Low energy structures of $(\mathrm{MgO})_{n}$ clusters with $n=3 \ldots 16$.

\section{Comparison to previous IR-REMPI experiments}

For a single size, $(\mathrm{MgO})_{15}$, the IR-UV two-color ionization spectrum can be compared to data obtained previously via IR-REMPI. ${ }^{31}$ The two experimental spectra are shown in Fig. 6 and appear rather different, with the IR-REMPI spectrum lacking any features below $600 \mathrm{~cm}^{-1}$ and showing a significant shift of $\sim 50 \mathrm{~cm}^{-1}$ for the most intense band. The shift would even slightly increase-the calculation predicts an additional shift of up to $11 \mathrm{~cm}^{-1}$-considering that the IR-REMPI spectra have been measured using $\mathrm{Mg}$ with the natural isotopic distribution $\left({ }^{24} \mathrm{Mg}: 79 \% ;{ }^{25} \mathrm{Mg}: 10 \% ;{ }^{26} \mathrm{Mg}: 11 \%\right)$, while we used ${ }^{26} \mathrm{Mg}$.

While the current experiments give clear evidence that the $(\mathrm{MgO})_{15}$ cluster has a hexagonal tube structure, the strong similarities between the IR-REMPI spectra of $(\mathrm{MgO})_{15}$ as well as of larger clusters and the phonon spectrum of bulk $\mathrm{MgO}$ led to the conclusion that cube like structures were observed. The lowest energy isomer of $(\mathrm{MgO})_{15}$ with a cubic sub-structure identified in our calculations is found to be $\sim 70 \mathrm{~kJ} \mathrm{~mol}^{-1}$ above the tubular ground-state (see $\mathrm{ESI} \dagger$ for structure and vibrational spectrum). At higher temperatures, required for IR-REMPI, the energetic ordering might change but this has not been observed in our calculations. Not only the predicted IR spectrum of that structure, but also those of other cubic sub-structures do not fit the IR-REMPI spectrum. The differences in the experimental spectra may be related to the specifics of the IR-REMPI process. The IR-REMPI spectrum can be significantly affected by (cross-)anharmonicities, leading, e.g. to large redshifts and changes in the IR intensities. ${ }^{55,56}$ Furthermore, the lack of spectral features at lower wavenumbers is more often observed in IR-REMPI spectra, ${ }^{45}$ as the thermionic emission of an electron requires the absorption of several hundred IR photons, and that cannot be easily fulfilled in the far IR. In principle, the IR-REMPI spectrum can be modeled, but this requires knowledge not only of the linear absorption spectrum but also detailed information on the anharmonicities. ${ }^{55,56}$

For larger clusters, the appearance of the cubic motif of the $\mathrm{MgO}$ bulk can be expected and experimental as well as theoretical evidence for this transition has been found. All previous experiments on $\mathrm{MgO}$ clusters showed a considerable change in the mass spectral abundance pattern for larger sizes. ${ }^{12,31}$ Furthermore, starting from $(\mathrm{MgO})_{21}$ IR bands close to frequencies of the perpendicular and parallel bulk phonon modes have been observed, whereas for the smaller $(\mathrm{MgO})_{15}$ cluster only a single feature was seen. ${ }^{31}$ Theory also predicts the structural change to occur just above the sizes considered here. For the $(\mathrm{MgO})_{3 \times 6}$ cluster the cubic motif is predicted to be energetically favored over the hexagonal tube structure. ${ }^{20}$ Further experimental and theoretical investigations will be necessary to locate the size-range where this transition occurs.

\section{Conclusions}

We have shown that neutral magnesium oxide clusters containing up to $15 \mathrm{MgO}$ units exhibit structures very different from the geometry of the simple cubic $\mathrm{MgO}$ bulk phase, with the only exception of $(\mathrm{MgO})_{4}$. The combination of gas-phase IR spectroscopy and density functional theory revealed unambiguously that the hexagonal ring is the fundamental building block for these $\mathrm{MgO}$ clusters. Fig. 7 summarizes the identified low energy structures.

Hexagonal tubes or rings are formed by clusters of $(\mathrm{MgO})_{3 k}$ composition with $k=1-5$, while the intermediate sizes prefer cage like structures. Also these cages incorporate the hexagonal ring motif. The structural transition towards the bulk-like cubic nano-crystals was not observed in the size range considered here. The cubic $(\mathrm{MgO})_{4}$ cluster is the only size with a global minimum structure close to the bulk geometry, however, for this size also 
higher lying isomers need to be considered to fully explain the experimental spectrum. For the $(\mathrm{MgO})_{13}$ cluster, the second and third lowest energy structures appear like distorted fragments of the bulk and some evidence for their presence was found, but also in this case the cage-like global minimum structure largely explains the experimental spectrum.

The here implemented ionization scheme does not rely on the formation of a messenger complex, as many photodissociation experiments do. The IR-UV two-color ionization technique is generally applicable and can be used to measure cluster-size specific IR spectra for many other metal oxides, and also pure metal clusters. In combination with tunable (vacuum) UV sources, this may make it possible to obtain vibrational spectra even down to the terahertz regime, as the UV photon energy can be chosen very close to the ionization threshold.

\section{Acknowledgements}

We gratefully acknowledge the support of the Stichting voor Fundamenteel Onderzoek der Materie (FOM) in providing beam time on FELIX. The authors thank the FELIX staff for their skillful assistance, in particular R. van Buuren, Dr B. Redlich and Dr A. F. G. van der Meer. The research leading to these results has received funding from the European Community's Seventh Framework Programme (FP7/2007-2013) under grant agreement no. 226716. This work is supported by the Cluster of Excellence "Unifying Concepts in Catalysis" coordinated by the Technische Universität Berlin and funded by the Deutsche Forschungsgemeinschaft. KK thanks the International Max Planck Research School "Complex Surfaces in Materials Science" for a fellowship.

\section{References}

1 J. A. Fagan, J. R. Simpson, B. J. Bauer, S. H. D. Lacerda, M. L. Becker, J. Chun, K. B. Migler, A. R. H. Walker and E. K. Hobbie, J. Am. Chem. Soc., 2007, 129, 10607-10612.

2 J. Kong, N. R. Franklin, C. W. Zhou, M. G. Chapline, S. Peng, K. J. Cho and H. J. Dai, Science, 2000, 287, 622-625.

3 P. G. Collins, K. Bradley, M. Ishigami and A. Zettl, Science, 2000, 287, 1801-1804.

4 K. Komatsu, M. Murata and Y. Murata, Science, 2005, 307, 238-240.

5 Y. B. Li, Y. Bando, D. Golberg and Z. W. Liu, Appl. Phys. Lett., 2003, 83, 999-1001.

6 M. S. Arnold, P. Avouris, Z. W. Pan and Z. L. Wang, J. Phys. Chem. B, 2003, 107, 659-663.

7 G. X. Wang, J. S. Park, M. S. Park and X. L. Gou, Sens. Actuators, B, 2008, 131, 313-317.

8 C. N. R. Rao, F. L. Deepak, G. Gundiah and A. Govindaraj, Prog. Solid State Chem., 2003, 31, 5-147.

9 P. D. Yang and C. M. Lieber, J. Mater. Res., 1997, 12, 2981-2996.

10 J. Zhan, Y. Bando, J. Hu and D. Golberg, Inorg. Chem., 2004, 43, $2462-2464$.

11 Q. Yang, J. Sha, L. Wang, Y. Wang, X. Ma, J. Wang and D. Yang, Nanotechnology, 2004, 15, 1004-1008.

12 P. J. Ziemann and A. W. Castleman, J. Chem. Phys., 1991, 94, 718-728.

13 T. M. Köhler, H. P. Gail and E. Sedlmayr, Astron. Astrophys., 1997, 320, 553-567.

14 E. de la Puente, A. Aguado, A. Ayuela and J. M. Lopez, Phys. Rev. B: Condens. Matter, 1997, 56, 7607-7614.

15 M. Wilson, J. Phys. Chem. B, 1997, 101, 4917-4924.

16 F. Calvo, Phys. Rev. B: Condens. Matter, 2003, 67, 161403.

17 J. S. Bhatt and I. J. Ford, Mon. Not. R. Astron. Soc., 2007, 382, 291-298.
18 C. Roberts and R. L. Johnston, Phys. Chem. Chem. Phys., 2001, 3, 5024-5034.

19 J. Carrasco, F. Illas and S. T. Bromley, Phys. Rev. Lett., 2007, 99, 235502.

20 R. B. Dong, X. S. Chen, X. F. Wang and W. Lu, J. Chem. Phys., 2008, 129, 044705.

21 Y. Zhang, H. S. Chen, B. X. Liu, C. R. Zhang, X. F. Li and Y. C. Wang, J. Chem. Phys., 2010, 132, 194304.

22 J. M. Recio, R. Pandey, A. Ayuela and A. B. Kunz, J. Chem. Phys., 1993, 98, 4783-4792.

23 M. J. Malliavin and C. Coudray, J. Chem. Phys., 1997, 106, 2323-2330.

24 G. Bilalbegovic, Phys. Rev. B: Condens. Matter, 2004, 70, 045407.

25 A. Jain, V. Kumar, M. Sluiter and Y. Kawazoe, Comput. Mater. Sci., 2006, 36, 171-175.

26 L. Chen, C. Xu and X. F. Zhang, THEOCHEM, 2008, 863, 55-59.

27 L. Chen, C. Xu, X. F. Zhang, C. A. Cheng and T. Zhou, Int. J. Quantum Chem., 2009, 109, 349-356.

28 K. Doll, J. C. Schön and M. Jansen, J. Chem. Phys., 2010, 133, 024107.

29 K. Asmis, A. Fielicke, G. von Helden and G. Meijer, Atomic Clusters: From Gas Phase to Deposited (The Chemical Physics of Solid Surfaces), Elsevier Science, 2007, vol. 12, pp. 327-375.

30 L. Andrews and J. T. Yustein, J. Phys. Chem., 1993, 97, 12700-12704.

31 D. van Heijnsbergen, G. von Helden, G. Meijer and M. A. Duncan, J. Chem. Phys., 2002, 116, 2400-2406.

32 K. R. Asmis, M. Brümmer, C. Kaposta, G. Santambrogio, G. von Helden, G. Meijer, K. Rademann and L. Wöste, Phys. Chem. Chem. Phys., 2002, 4, 1101-1104.

33 A. Fielicke, G. Meijer and G. von Helden, J. Am. Chem. Soc., 2003, 125, 3659-3667.

34 K. R. Asmis, G. Meijer, M. Brümmer, C. Kaposta, G. Santambrogio, L. Wöste and J. Sauer, J. Chem. Phys., 2004, 120, 6461-6470.

35 K. R. Asmis and J. Sauer, Mass Spectrom. Rev., 2007, 26, 542-562.

36 M. Sierka, J. Döbler, J. Sauer, G. Santambrogio, M. Brümmer, L. Wöste, E. Janssens, G. Meijer and K. R. Asmis, Angew. Chem., Int. Ed., 2007, 46, 3372-3375.

37 A. Kirilyuk, A. Fielicke, K. Demyk, G. von Helden, G. Meijer and T. Rasing, Phys. Rev. B: Condens. Matter, 2010, 82, 020405.

38 K. Kwapien, M. Sierka, J. Döbler, J. Sauer, M. Haertelt, A. Fielicke and G. Meijer, Angew. Chem., Int. Ed., 2011, 50, 1716-1719.

39 M. Putter, G. von Helden and G. Meijer, Chem. Phys. Lett., 1996, 258, 118-122.

40 A. Fielicke, J. T. Lyon, M. Haertelt, G. Meijer, P. Claes, J. de Haeck and P. Lievens, J. Chem. Phys., 2009, 131, 171105.

41 A. Fielicke, G. von Helden and G. Meijer, Eur. Phys. J. D, 2005, 34, 83-88.

42 S. E. Stein and B. Rabinovitch, J. Chem. Phys., 1973, 58, 2438-2445.

43 V. N. Bagratashvili, V. S. Letokhov, A. A. Makarov and E. A. Ryabov, Laser Chem., 1984, 4, 171-274.

44 D. Oepts, A. F. G. van der Meer and P. W. van Amersfoort, Infrared Phys. Technol., 1995, 36, 297-308.

45 G. von Helden, D. van Heijnsbergen and G. Meijer, J. Phys. Chem. A, 2003, 107, 1671-1688.

46 TURBOMOLE V6.3 2011, a development of University of Karlsruhe and Forschungszentrum Karlsruhe GmbH, 1989-2007, TURBOMOLE $\mathrm{GmbH}$, since 2007; available from http://www.turbomole.com.

47 M. Sierka, Prog. Surf. Sci., 2010, 85, 398-434.

48 A. D. Becke, J. Chem. Phys., 1993, 98, 5648-5652.

49 C. T. Lee, W. T. Yang and R. G. Parr, Phys. Rev. B: Condens. Matter, 1988, 37, 785-789.

50 A. Schäfer, C. Huber and R. Ahlrichs, J. Chem. Phys., 1994, 100, 5829-5835.

51 K. Eichkorn, F. Weigend, O. Treutler and R. Ahlrichs, Theor. Chem. Acc., 1997, 97, 119-124.

52 F. Weigend and R. Ahlrichs, Phys. Chem. Chem. Phys., 2005, 7, $3297-3305$.

53 M. Sierka, A. Hogekamp and R. Ahlrichs, J. Chem. Phys., 2003, 118, 9136-9148.

54 C. Ochsenfeld and R. Ahlrichs, Ber. Bunsen-Ges. Phys. Chem., 1994, 98, 34- 47.

55 G. von Helden, I. Holleman, G. Meijer and B. Sartakov, Opt. Express, $1999,4,46-52$

56 J. Oomens, A. G. G. M. Tielens, B. G. Sartakov, G. von Helden and G. Meijer, Astrophys. J., 2003, 591, 968-985. 